Revista Científica General José María Córdova

(Revista colombiana de estudios militares y estratégicos)

Bogotá D.C., Colombia

ISSN 1900-6586 (impreso), 2500-7645 (en línea)

Web oficial: https://www.revistacientificaesmic.com

\title{
La medicina clásica en la sanidad militar de la Campaña Libertadora de Nueva Granada 1819
}

\section{Erika Constanza Figueroa Pedreros}

https://orcid.org/0000-0001-6241-344X

erika.figueroa@esmic.edu.co

Escuela Militar de Cadetes “General José María Córdova”, Bogotá D.C., Colombia

Citación: Figueroa Pedreros, E. C. (2019). La medicina clásica en la sanidad militar de la Campaña Libertadora de Nueva Granada 1819. Revista Científica General José María Córdova, 17(27), 645-662. http://dx.doi.org/10.21830/19006586.486

Publicado en línea: 1 de julio de 2019

Los artículos publicados por la Revista Científica General José María Córdova son de acceso abierto bajo una licencia Creative Commons: Atribución - No Comercial - Sin Derivados.

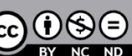

Para enviar un artículo:

https:/www.revistacientificaesmic.com/index.php/esmic/about/submissions
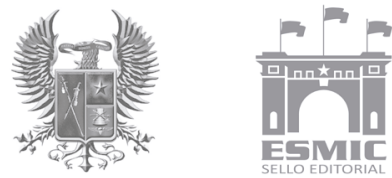


\section{La medicina clásica en la sanidad militar de la Campaña Libertadora de Nueva Granada 1819*}

\section{Classical medicine in military health in the Nueva Granada Liberating Campaign of 1819}

\section{Erika Constanza Figueroa Pedreros}

Escuela Militar de Cadetes “General José María Córdova”, Bogotá D.C., Colombia

Resumen. Este artículo muestra cuáles fueron los fundamentos de la medicina hipocrática y galena del mundo clásico que convergieron en el contexto histórico de la sanidad militar en la Campańa Libertadora de la Nueva Granada de 1819. El aporte teórico corresponde específicamente a la caracterización del diagnóstico, el tratamiento y la relación entre el médico y el enfermo, evidenciados tanto en los escenarios de guerra como en los casos de enfermedad común. Por tanto, la pretensión del documento es identificar las fuentes clásicas de la medicina griega y romana en un hito histórico de gran importancia en el marco de la conmemoración del Bicentenario de la Batalla del Puente de Boyacá.

Palabras ClaVe: Campaña Libertadora; ciencias médicas; historia antigua; historia nacional; profesión médica; sanidad militar

AвSTRACT. This article presents the foundations of Hippocratic and Galenic medicine of the classical world that converged in the historical context of military health in the Liberating Campaign of New Granada of 1819. The theoretical contribution is the characterization of diagnosis, treatment, and the relationship between the doctor and the patient evidenced both in war scenarios and cases of common illness. Therefore, the document aims to identify the classical sources of Greek and Roman medicine in a historical milestone of great importance in the framework of the commemoration of the Bicentennial of the Battle of the Boyacá Bridge.

KeYwords: ancient history; Liberating Campaign; medical profession; Medical Sciences; military health; national history

Sección: Dosier Bicentenario • Artículo de investigación científica y tecnológica

Recibido: 2 de mayo de 2019 • Aceptado: 14 de junio de 2019

\footnotetext{
* La base histórica de este documento está en otro texto de la autora ("La sanidad militar en la Campańa Libertadora de la Nueva Granada en 1819"), que fue publicado por la Revista Histórica del Ejército Nacional en su edición n. ${ }^{\circ} 1$ (diciembre de 2013, Centro de Estudios Históricos del Ejército, pp. 26-37).
}

ConTACto: Erika Constanza Figueroa Pedreros $\bigotimes$ erika.figueroa@esmic.edu.co 


\section{Introducción}

Los estudios de la medicina clásica han tomado rumbos interesantes en cuanto al origen de las enfermedades, la profesión médica, los tratamientos y la relación entre el enfermo y el médico; Grecia y Roma son el ejemplo paradigmático. Autores como Laín Entralgo (1964, 1978) y Gozalbes Cravioto y García García han abordado el tema y han explicado los aspectos mencionados conforme a la medicina hipocrática y galena, elementos que sirven para relacionar los aspectos médicos de la época clásica con la medicina moderna del siglo XIX.

A su vez, la Campaña Libertadora de la Nueva Granada ha sido uno de los acontecimientos históricos más importantes y analizados a nivel regional, nacional e internacional, especialmente, en este 2019. Este evento histórico consolidó destacadas acciones militares del Ejército patriota en contra del Ejército realista, lo cual permitió la continuación del proyecto republicano, promulgado años atrás, hasta su consolidación. Sin embargo, son muchos los temas en este contexto que merecen especial atención por los investigadores, uno de ellos es el de la sanidad militar en la Campaña Libertadora de la Nueva Granada y la posible manifestación de la medicina griega y romana, identificada en el uso de las fuentes clásicas.

En las investigaciones encontradas referentes a la sanidad militar en la Campaña Libertadora de 1819, se ha ignorado el tema de la conexión histórica entre las fuentes de la medicina clásica y su impacto en el cuerpo médico de los ejércitos de la época. Si bien los balances historiográficos apuntan a la descripción detallada y al análisis en profundidad de la sanidad militar desde finales del siglo XVIII hasta el inicio del proyecto de la Gran Colombia (Otálora Cascante, 2017), son escasos los autores que intentan entrelazar la herencia de la medicina griega y romana con los tratamientos y la relación médico-enfermo en el contexto de la guerra. Así, este documento busca responder al cuestionamiento dirigido a caracterizar las fuentes clásicas de la medicina griega y romana en la sanidad militar de la Campaña Libertadora de la Nueva Granada de 1819.

A partir de esta pregunta-problema, la hipótesis planteada apunta a demostrar la existencia de una relación entre la medicina hipocrática y galena en el contexto médico de la sanidad militar evidenciada en la Campaña Libertadora de la Nueva Granada de 1819, entendida como la práctica —en parte- de algunos fundamentos médicos propios de las fuentes clásicas del siglo V a. C. y I d. C., vistos, especialmente, en el escenario bélico de contienda debido al establecimiento de un proyecto republicano en contra de una monarquía ya instaurada, en las primeras décadas del siglo XIX.

Ahora bien, corresponde cerrar este apartado con la definición de la medicina hipocrática y galena, seguida de la de sanidad militar en ambiente de campaña. Señala Laín Entralgo (1964, p. 29) que la medicina griega permitió un proceso "técnico" entre el médico y el enfermo, entendido como el descubrimiento de dos elementos clave para tratar las enfermedades: el qué y el por qué: ¿qué enfermaba a la gente? y ¿por qué se daban las 
enfermedades? Esto se sumaba a la preparación empírica de los médicos helenísticos que, con el paso del tiempo y debido a la invasión romana al Peloponeso, se trasladaron a la metrópoli con el fin de diagnosticar y mejorar en el arte de curar.

Por último, según la Real Academia de la Lengua Española, sanidad militar es un "cuerpo de profesores médicos, farmacéuticos y veterinarios y de tropas especiales, que prestan sus servicios profesionales a los ejércitos de aire, mar y tierra" (sanidad militar, 2001). Ahora bien, para la época de la Campaña Libertadora, los médicos eran muy pocos, debido a las restricciones al sistema educativo, tanto así que los llamados boticarios pertenecían también al selecto grupo de quienes recetaban remedios naturales y acompañaban a los cuerpos militares en campaña (Roselli, 1979).

Las fuentes consultadas para este escrito no refieren que los comandantes de las divisiones por parte del Ejército Libertador mencionaran el término sanidad en su esencia, solamente conceptos como heridos, moribundos, hospitales y remedios. No obstante, podríamos señalar que la sanidad militar en las primeras décadas del siglo XIX hacía referencia a los factores siguientes (Fundación para la Conmemoración del Bicentenario del Natalicio y el Sesquicentenario de la Muerte del General Francisco de Paula Santander, 1988):

- Primero: no dejar morir a los combatientes que sufrieran heridas de gravedad, cuidar de ellos en lugares alejados de las contiendas y en caso extremo impartir los sagrados sacramentos. Las personas encomendadas para cumplir con tal misión solían ser uno o dos médicos cuando había presupuesto, varios religiosos y auxiliares.

- Segundo: de los recursos económicos otorgados a los ejércitos, apartar cierta cantidad con el fin de comprar lo necesario en la dotación de los hospitales de refugio a militares o buscar asilo en casas de los pueblos.

- Tercero: procurar que el lugar de descanso en zonas inhóspitas, después de las largas jornadas, contara con las mínimas condiciones para poder continuar con el camino. Como se verá más adelante, este fue un asunto de bastante consideración por parte del general Santander, quien comandaba la División de Vanguardia del Ejército Libertador.

\section{La teoría de la medicina clásica y las corrientes teóricas médicas a principios del siglo XIX}

Desde el enfoque teórico, la medicina clásica emprendió un camino más técnico y especializado en el conocimiento de las enfermedades, del hombre y su naturaleza (physis), conocimiento manifestado en la relación que debía existir entre el médico y el enfermo. Laín Entralgo, experto en el conocimiento histórico de la medicina en el mundo, profundizó sus estudios en caracterizar esas "vicisitudes que la relación entre el médico y el enfermo han experimentado en la historia del mundo occidental" (1964, p. 29). Si bien, 
son varios los fundamentos teóricos extraídos de la medicina hipocrática y galena, el autor enfatiza en que "la amistad del enfermo con el médico consiste en la confianza anhelante y agradecida del inválido, en quien puede devolverle su validez normal [...] es una confianza pensada en dos momentos: la confianza del enfermo en la medicina y la confianza en el médico que va a tratar la enfermedad" (1964, p. 54).

Heredera de la medicina hipocrática, la medicina galena estuvo enfocada en considerar el médico como un sabio, con plena voluntad para entender la enfermedad y, mediante el diagnóstico, conseguir la admiración del enfermo y quienes lo acompañaban (Laín, 1978, p. 119). "En la medicina romana, el debate y la crítica se hicieron más visibles y, a su vez, se contó con un desarrollo considerable del concepto sanidad pública, que tuvo su núcleo básico en la higiene y en la cultura del agua" (Gozalbes Cavrioto \& García García, 2010, p. 334). Igualmente, en el contexto de guerra, también fue de gran aporte el desarrollo de la sanidad militar: los expertos en el tema afirman que, debido a las campañas militares en la Roma republicana — guerras púnicas—, la guerra civil en la época del triunvirato de Julio César y en la Roma imperial de Trajano, Marco Aurelio y Septimio Severo, fue posible salvar muchas vidas, gracias a la oportuna reacción de los generales para incluir en las legiones médicos o galenos.

Frente a este panorama de la medicina clásica, para el año de 1819 en el territorio de la Nueva Granada existieron dos corrientes teóricas de la "ciencia médica": la corriente francesa y la corriente inglesa. La primera era reconocida por su "amplia influencia en Europa y en todo el mundo durante las dos primeras décadas del siglo XIX" (Miranda Canal, 1992, p. 3). Su importancia radicó en el uso que hizo de ella el Ejército realista no solo en situaciones de combate, sino en la instalación de escuelas de medicina, hechos acontecidos durante la permanencia de Juan Sámano en el poder, quien, a finales de 1816, creó una academia de medicina por la falta de figuras médicas formadas para la atención de los ejércitos. Obligó a los médicos residentes en Santafé a reunirse una vez al mes para perfeccionar sus conocimientos científicos, bajo la dirección del médico español Fernández de la Reguerra (Otálora Cascante, 2010, p. 124)

La difusión de las doctrinas francesas en la segunda década del siglo XIX tuvo contradictores, en especial, quienes estaban orientados por las doctrinas inglesas. Precisamente estas últimas llegaron a Nueva Granada gracias a la Legión Británica, acompañantes de primera mano de la Campaña Libertadora de Bolívar. Las diferencias entre las dos corrientes estaba en las concepciones de las enfermedades, el tratamiento hacia los enfermos y los "medicamentos" usados. Puesto que la teoría francesa concebía las enfermedades como respuesta al exceso de irritación (enfermedades esténicas), con base en ello promulgaba remedios terapéuticos, de los cuales la sangría era el eje principal (Miranda Canal, 1992). 


\section{Analogía de los tratamientos médicos en el periodo clásico y los tratamientos médicos en el siglo XIX}

La fuente clásica de la medicina hipocrática (Corpus Hippocraticum) comprende 58 escritos divididos en 73 libros, cuya edición más conocida es la de Littré, en París (1839). Laín Entralgo $(1964,1970)$ hace al respecto un importante análisis, en especial, en lo relacionado con la naturaleza del hombre, los aspectos clínicos, patológicos, terapéuticos y la deontología en la medicina técnica.

Los escritos hipocráticos, en general, estaban enfocados en la sensibilidad del cuerpo y sostenían que el diagnóstico a partir de los sentidos y el tratamiento realizado por el médico eran los fundamentos "técnicos-fisiológicos" para curar las enfermedades (katástasis) en un entorno llamado naturaleza. A esto se sumaba otro factor de gran relevancia, el vínculo de amistad producido por la constante interacción entre el médico y el enfermo (Laín Entralgo, 1964, p. 79). El arte de curar estaba condicionado al conocimiento que tenía el médico de la naturaleza y del hombre, es por ello que, en el pensamiento griego, había dos tipos de tratamiento: las dietas idealizadas de una medicación farmacoterápica y la cirugía basada en dos elementos, a saber, hierro y fuego (Laín Entralgo, 1964, p. 82).

Los tratamientos médicos en Roma lograron transformar, en parte, la concepción de la medicina griega en relación con el cuerpo y las enfermedades, los diagnósticos y los tratamientos. Galeno, en especial, "señalaba que el tratamiento, además de los fármacos, debía estar acompañado de la higiene, gimnasia, ejercicios respiratorios y dieta. A diferencia de Hipócrates, creía que los fármacos eran parte importante del tratamiento, por ello recomendaba el empleo de vegetales, minerales y sustancias de origen animal" (Gargantilla, 2011, p. 94).

Comparado con el siglo XIX, los tratamientos del mundo clásico no diferían totalmente del mundo occidental moderno, tanto en el entorno de enfermedad natural en área urbana o área rural como en situaciones complejas de salud debido a la participación directa o indirecta de las acciones de armas. En cualquiera de los casos, los médicos propendían por salvar la vida del enfermo o paciente.

En el mundo moderno de las primeras décadas del siglo XIX, los expertos en el tema afirman que la mayoría de los tratamientos médicos usados para la cura de las enfermedades tropicales, de bajas temperaturas y las heridas adquiridas durante las batallas eran de origen natural. Los tratamientos más usuales fueron sangrías, purgas, enemas (lavados) y ungüentos (Otálora Cascante, 2010). En tiempos de guerra, la amputación solía convertirse en la "salvación" de la persona, no obstante, en varias ocasiones moría desangrada a causa del procedimiento y de los pocos cuidados.

El constante uso de las plantas fue vital para la preparación de las purgas, los enemas y ungüentos. La creencia popular de la sanación teniendo en cuenta el olor, tamańo y aspecto de la planta contribuía a la formación del imaginario colectivo y las tradiciones culturales que eran difundidas mediante tradición oral de generación en generación. Algunos de los tratamientos de los cuales se tiene conocimiento son los siguientes: 
Ungüento eficacísimo para curar heridas asi de hierro como de balas y también para apostemas, tumores y llagas

Media libra de aceite de comer, tres y media onzas de albayalde (carbonato de plomo), una onza de mirra, tres onzas de cera nueva blanca. Se pone el aceite en una cazuela y en estado hirviendo se pone el albayalde sin cesar de menear y empezando a hervir se ha de poner la mirra, y luego poner la cera hasta que se cuaje, sin dejar de menear; sacarlo y guardarlo que se conservan unos ańos. Se aplica con parche de piel y si la herida pasa de parte en parte, enjuagando bien la sangre se pone un parche a cada agujero y no se necesita de otra curación mientras la herida no sea mortal. (Díaz Piedrahita \& Mantilla, 2002, p. 71)

\section{Recetas para la quemadura del fuego o del agua caliente}

Aplicar tierra mojada con agua, o barro colorado y deshecho en agua o untarse mantequilla, aplicar lentejuela del agua, culantro verde medio cocido o acelgas. Cuando se levantan las ampollas, no abrirlas sino hasta el tercer o cuarto día, pasándolas por agua. Abiertas las ampollas, tome media onza de cal lavada en varias aguas y con aceite rosado usar como ungüento y untar en las ampollas con una pluma o aplicar estiércol de res con leche mezclado o sin ella. (Díaz Piedrahita \& Mantilla, 2002, pp. 90-91)

\section{Ungüento para llagas inflamadas}

Pez griega, trementina de frailejón, tuétano de vaca y aceite, tanto de lo uno como de lo otro por iguales partes, cuajarlo al fuego manso con cera blanca, enjudía de gallina y manteca de vaca sin sal. (Díaz Piedrahita \& Mantilla, 2002, p. 98)

Señala Otálora Cascante (2010) que el ejército comandando por Morillo sufrió el mal de llagas constantemente, entre 1818 y 1819, en la travesía por los Llanos Orientales y en tierras de la provincia de Tunja.

\section{Para purgación}

Cebolla cabezona blanca, rajarla en distintas partes y aquellas rajaduras llénelas de vino, y ponla al sereno y que vaya aquel vino goteando en una vasija y a la mañana siguiente beberlo.

Una libra de quinua, dos veces bien lavada, ponla al sol que se seque, muélela, pásala por un caldero blanco y vuélvalo a poner al sol con una libra de azúcar revuelta. Volver a secar bien, y cocinar bien la raíz de la zarzamora, en esta agua mañana y tarde, hacer zarzamora mañana y tarde con la quina y azúcar y tomar. (Díaz Piedrahita \& Mantilla, 2002, p. 104)

\section{Ungüento para llagas}

El zumo de verbena que llaman Rabo de Alacranes, mezclado con un polvo de raíz de lirio cárdeno, mezclado con miel de abejas modifica todo género de llagas y cubre la carne de los huesos descarnados. (Díaz Piedrahita \& Mantilla, 2002, p. 106) 
Tome cuatro onzas de mantequilla fresca sin sal, lávala tres veces y agrégale una onza del aceite de almendras dulces, el peso de un real de planta de piedra lipiz y un poco de aluminio de Castilla; cada cosa de por sí bien quemada y luego bien remolidas, lo agregarás con la mantequilla y aceite de almendras dulces y con esto harás un ungüento y con parche lo pondrás dos veces al día sobre la llaga. (Díaz Piedrahita \& Mantilla, 2002, p. 131)

Cada uno de los componentes descritos anteriormente y sus preparaciones eran usados por los boticarios, curanderos, auxiliares y algunos médicos titulados; como era de esperarse, ante las condiciones dadas por la guerra, las necesidades logísticas en cuanto al almacenamiento de ciertos elementos para realizar los tratamientos era de gran trascendencia, porque, al realizar a tiempo algún procedimiento, podían salvarse aquellos soldados con heridas no tan graves originadas por el combate o, en su defecto, con enfermedades causadas por los cambios climáticos producto de la campaña militar.

En 1818 y 1819, las fuentes documentales arrojan un gran número de casos relacionados con los tratamientos médicos improvisados para salvar la vida de los soldados, algunos de ellos para las picaduras de los mosquitos y serpientes en territorio selvático o pantanoso. Según Otálora Cascante (2017), en el relato del médico de la Legión Británica, al arribar a la Capitanía General de Venezuela

en las bocas del Orinoco [este] contó cómo los nativos se pintaban el cuerpo con una semilla roja que él denominó Roocoo o Ruco, la que los protegía de la picadura de las serpientes e insectos, que según él se contaban por millones y que le causaron numerosas incomodidades. (p. 298)

Ahora bien, en respuesta al significado de sanidad dado por la Real Academia de la Lengua Española, frente a la teoría médica clásica contrastada por Laín $(1964,1978)$ y por Gargantilla (2011) con la teoría moderna del siglo XIX, resulta interesante identificar la posible relación entre el funcionamiento de la sanidad militar en ambos ejércitos - estructura y organización - y los fundamentos de la medicina clásica — poniendo énfasis en las fuentes grecorromanas de la medicina antigua-. Tal es el ejercicio que se desarrolla a continuación.

\section{La sanidad en los ejércitos del rey}

En cualquier teatro de operaciones, "la atención médica oportuna dependía de la capacidad de los jefes militares para dirigir las contiendas, la calidad de los cirujanos y médicos, disponibilidad de los alimentos, ropa, suministros médicos y recursos financieros" (Romero, 1996, p. 37). El bando realista tuvo preocupación, en cierta medida, por acondicionar los elementos básicos requeridos, en mejora de la salud de sus combatientes. Por ejemplo, el personal que trabajó en los hospitales creados por las condiciones de guerra ayudó a los soldados, desde los tiempos de la Reconquista hasta el inicio de la República 
(desafortunadamente en muchas ocasiones era demorado el pago por falta de presupuesto para esa empresa).

Este panorama del mundo moderno, comparado con la medicina romana de la Antigüedad, tiene algunas diferencias, pues mientras en la sanidad militar de los primeros ańos de la República era precario el pago de sueldos, en la Roma imperial las condiciones de estabilidad de los médicos extranjeros mejoraron, en especial, en época de Adriano. Los médicos extranjeros debían prestar servicio militar obligatorio, lo cual indicaba que las legiones contaban con personal de asistencia para las enfermedades o heridas causadas por la guerra (Gargantilla, 2011).

En ocasiones, los comandantes del Ejército Español no siempre contaban con la colaboración de los auxiliares en salud; de forma que perseguían y obligaban a los pocos médicos que habitaban las ciudades a incorporarse a la causa pacifista en pro de la salvación de la tropa:

... algunos como Manuel María Quijano, el Doctor Domínguez, y el Doctor Flórez en la provincia de Popayán fueron sometidos a presidios y torturas. Otros fueron forzados a prestar sus servicios profesionales a los militares españoles: como el caso de José Félix Merizalde, José Zapata, Juan Gualberto Gutiérrez, Santos González, Benito Osorio y Pedro Lasso de la Vega. (Quevedo, 2008, p. 116)

Quienes han estudiado este tema mencionan que, mientras los españoles promulgaban normalidad social y política, "sin descuidar lo sanitario y médico, la guerra de guerrillas continuaba. Los ejércitos republicanos se estructuraban poco a poco, recomponiendo lentamente sus líneas de mando, estructurando la estrategia y determinando sus tácticas de batalla; no obstante, las enfermedades atacaban a unos y otros". (Quevedo, 2008, p. 121).

En campaña, el personal médico esperaba en los pueblos más cercanos para disponer de lo necesario en la curación de los enfermos. Según Cayo Leonidas Peñuela (1919), después de la Batalla del Pantano de Vargas, "Barreiro, acuarteló sus tropas en la población de Paipa y apenas separó medio Batallón del Rey para situarlo, como cuerpo de observación, en la Lomita, se preocupó primero en el cuidado de los heridos, que eran más de cientos y en reorganizar los batallones" (p. 308). No fue posible encontrar información que registrara el acompañamiento de los médicos al servicio realista en el escenario de batalla, sin embargo, es un interrogante histórico que puede ser fruto de futuras investigaciones.

Conforme pasaban los días para darse el desarrollo de las contiendas bélicas en 1818 y 1819, el Ejército español sufrió las inclemencias del clima de una manera abrupta y compleja. Si bien organizaron hospitales de campaña, en las áreas de difícil acceso (como los Llanos Orientales de Venezuela y de Nueva Granada) no fueron posibles los diagnósticos y los tratamientos para el buen desenvolvimiento de la ciencia médica tal como se tenía planeado. Ejemplo de ello fue el constante uso de los elementos de la naturaleza 
(Modo Tékhnêl) para preparar las pociones, ungüentos y brebajes en beneficio de la salud de los militares:

[...] la simple picada de un mosquito priva la vida de un hombre o se le origina una ulcera que después de tenerlo impedido mucho tiempo, le deja inutilizado. El dormir al sereno en los campamentos acaba de producir una multitud de ciegos en la División del brigadier Canterac. Los alimentos del país causan a los europeos enfermedades de toda especie y hay muy pocos que resistan su fatal influjo. Los inmensos desiertos en que se hace la guerra, la falta de auxilio de todo género, las aguas encharcadas que por precisión han de beberse en muchas ocasiones, y la fatiga extraordinaria del soldado en distancias tan considerables por estaciones tan diversas y variadas, todo contribuye a nuestra destrucción y a la aniquilación de las tropas. (Earle, 2014, p. 310)

\section{Conformación de la Tercera División}

Para comprender mejor el funcionamiento de la sanidad militar en el bando realista, se ha querido poner a consideración la información proporcionada por Otálora Cascante (2010), y así dar a conocer la conformación de la Tercera División del Ejército, comandada por el coronel José María Barreiro. Dicho cuerpo militar tuvo los más importantes enfrentamientos contra la tropa patriota, como la Batalla del Pantano de Vargas y la Batalla del Puente de Boyacá (tabla 1).

Tabla 1. Estado de la fuerza disponible del Ejército Expedicionario

\begin{tabular}{cllrr}
\hline $\begin{array}{c}\text { Ejército expedicionario } \\
\text { de Costafirme }\end{array}$ & \multicolumn{4}{c}{ Estado Mayor - Tercera División, Tunja, $\mathbf{5}$ de julio de 1819 } \\
\hline \multicolumn{1}{c}{ Armas } & Destinos & Cuerpo a que pertenece & Total & Caballos \\
\hline \multirow{4}{*}{ Infantería } & En Paya & I,II del Rey y del Tambo & 310 & \\
& En Puebloviejo & I del Rey & 66 & \\
& & I del Rey & 422 & \\
& En Tunja & II de Numancia & 384 & \\
Caballería & & Dragones de Granada & 149 & 156 \\
& En Sogamoso & Dragones de Granada & 227 & 198 \\
\hline & En Tunja & Total & $\mathbf{1 5 5 8}$ & $\mathbf{3 5 4}$ \\
\hline
\end{tabular}

Nota: Se incluyen en el total jefes, oficiales, sargentos, tambores y trompetas, cabos y soldados.

Fuente: Otálora Cascante (2017, p. 330).

1 Laín Entralgo menciona que existe un doble modo de comprender la dinámica de la naturaleza en la medicina griega: para que sea humanamente razonable, sus movimientos han de producirse sometidos a cierta necesidad (hay cosas que son pudiendo no haber sido necesarias). El otro punto son las cosas que no pueden ser y que se dan por suerte (Laín Entralgo, 1964, p. 39). 
El total de los batallones de infantería y caballería comandados por Barreiro no llegaba a los 2000 hombres. Cabe resaltar un aspecto importante para el objeto de estudio del texto: en la fuente consultada no se encontraron registros que verificaran el acompañamiento directo del personal médico en las batallas, lo que confirma aún más la posibilidad de hallar personal de asistencia o auxiliares en lugares alejados de las contiendas militares. "Los heridos de los ejércitos realistas fueron confiados a comandantes importantes de los escuadrones" (Peńuela, 1919, p. 139), quienes coordinaban el traslado a los hospitales de campaña.

Estas actuaciones fundamentan "la relación de amistad" entre el enfermo y el médico, pues en este último recaía la responsabilidad de salvarle la vida al combatiente al llegar al recinto: el médico debía "conocer e identificar el problema a la luz de la medicina técnica y organizar la curación a partir de los conocimientos fisiológicos del cuerpo" (Laín Entralgo, 1978, p. 60).

\section{La sanidad en el Ejército patriota}

En comparación con la historiografía del siglo XIX, pocos son los autores nacionales que se han interesado por la sanidad militar ${ }^{2}$. En el proceso de información para la escritura de este documento, se encontraron algunos hechos que confirman la intención de organizar un cuerpo médico de apoyo a la misión independentista, igual que en el Ejército realista y en el mundo clásico. Los relatos de quienes vivieron en aquel tiempo, las comunicaciones oficiales y secretas entre los comandantes de las divisiones y las diferentes fuentes secundarias permitieron establecer los inicios de la sanidad militar en la época republicana. Lo precario de las condiciones de salvaguardar la vida de los combatientes dificultaba el pleno ejercicio de las funciones médicas, y la geografía y el clima de la región hizo más difícil la localización de los enfermos y heridos. Debido a esas circunstancias fueron presos de muchas enfermedades como disentería, afecciones respiratorias, viruelas, tabardilla, tifoideas e hipotermias (Romero, 1996, p. 37-39).

Este tipo de diagnósticos en el contexto de guerra en tiempos modernos apunta a la

acción de establecer, instituir o constituir esa enfermedad o katástasis de la medicina clásica, seguida de una resolución o a veces dilema por parte del médico en clasificar al enfermo en qué tan sano o qué tan enfermo podía encontrarse, por último, era obligatorio emitir el diagnóstico e iniciar el mejor tratamiento conocido. (Laín Entralgo, 1964, p. 59).

2 En las tesis de maestría y de doctorado de Otálora $(2010,2017)$ se encuentran balances historiográficos de gran importancia para quienes estén interesados en conocer más sobre la sanidad militar en las primeras décadas del siglo XIX en Nueva Granada y Venezuela. Con respecto a la medicina clásica romana, el artículo de Gozalbes y García García (2010) es una excelente muestra de investigación sobre el tema. 
Otro factor de análisis fueron los médicos americanos que participaron en la Campaña Libertadora, quienes estuvieron dirigidos por los médicos y cirujanos de la Legión Británica. Ellos lograron con relativo éxito organizar en el Ejército una medicina militar eficiente (Romero, 1996).

Entre los médicos americanos podemos mencionar a José Joaquín García, Rafael Flórez, Joaquín Cajiao, José María Unday, Fray Antonio Macary, José María Fernández de Córdova, Miguel Domínguez, Esteban Quintana, Joaquín Durán, José María Valenzuela, José Fernández Madrid, Juan Pardo, José Joaquín Sánchez, Ignacio Durán, Isaac Calvo, Miguel Ibánez, Joaquín Moya, Juan Gualberto Gutiérrez, José García, Gregorio Posada, Francisco Antonio Mendoza, Jorge Vargas y Joaquín Maldonado; de la selecta lista, muy pocos sirvieron a la causa patriota. (Romero, 1996, p. 38)

Gracias a los médicos de la Legión Británica se implementaron algunos tratamientos que, unidos a los esfuerzos de los galenos nombrados, camilleros, enfermeros y esclavos, constituyeron un aporte significativo para Bolívar y su Ejército. Entre los médicos ingleses encontramos a los doctores Charles Moore, Hughes Blair, George Mayre, John Roberton —quien en 1818 fue nombrado director general de los Hospitales de las Provincias libres de Nueva Granada-. Estos doctores se quedaron a vivir en la nueva nación colombiana, así como el cirujano personal de El Libertador, Thomas Foley, quien debió amputarle el brazo al coronel James Rook después de la Batalla del Pantano de Vargas.

En contraste con la época romana imperial, Aulio Cornelio Celso afirmaba que entre las cualidades de todo cirujano debían contarse las siguientes:

ser joven o cuando menos no muy viejo, su pulso debe ser firme y seguro, sin que jamás le tiemble. Debe poder usar la mano izquierda con igual destreza que la derecha, su visión debe ser aguda y clara, su mente intrépida y debe sentir la piedad necesaria, no a tal grado que se sienta conmovido por las lágrimas, no debe ni apresurar la operación más de la cuenta, ni cortar menos de lo que fuere necesario, sino hacer todo exactamente como si los gritos del otro no le impresionaran... (Gargantilla, 2011, p. 89)

Desde la perspectiva de la enfermedad, por desconocimiento de la teoría de los gérmenes, asociado con la falta de comprensión sobre la transmisión de enfermedades e infecciones, los médicos del Ejército patriota y su equipo de trabajo usaban cualquier contaminante para resolver los problemas quirúrgicos presentados con la mayoría de los pacientes (Romero, 1996, p. 39). Fue una constante la urgencia de inyectar recursos económicos a la higiene, salubridad y mantenimiento de los hospitales y medicinas para los soldados en campaña. Tal como se evidenció con el Ejército del rey, las enfermedades producto de la (tykhê) necesidad de estar en guerra o la (anánkề) suerte de contraer alguna molestia en el cuerpo, como afirma Hipócrito, son escenarios que debía comprender el médico y, con base en ellos, establecer y fomentar esa philia médica o amistad con el enfermo (Laín Entralgo, 1964, p. 40). 


\section{Sanidad en la ruta libertadora}

Desde 1819, los mandos militares trataron de implementar algunos elementos que sirvieran para la protección de la tropa. En cartas fechadas en enero de ese año, dirigidas por el general Santander al Tesoro Público, este mencionaba la necesidad de administrar recursos y requerir medicamentos para los hospitales sin necesidad de autorización, con lo cual confirmaba que se trataba de un asunto bastante importante que no daba espera. Unos meses más adelante, ordenó entregar al comisario de guerra Antonio María Ramírez los recursos económicos suficientes para la subsistencia del hospital (Rodríguez Plata \& Lee López, 1971, pp. 244-245).

En reiteradas ocasiones, informaban al general Santander sobre el estado de la tropa, ejemplo de ello son las notificaciones de ciertos funcionarios y su crítica situación de salud (Rodríguez Plata \& Lee López, 1971, p. 276). O caso contrario, él mismo preguntaba por ellos dando órdenes claras para evacuar a los comandantes que estaban mal y no podían dirigir la tropa para la marcha (p. 359), como lo sucedido con el coronel Juan Galea, perteneciente a la división de la retaguardia, quien manifestó su deteriorado estado de salud (Peñuela, 1919, p. 218).

Las memorias de la época señalaban que "las largas caminatas, el clima de la región y las dificultades propias de la zona originaron que al iniciar el recorrido ya fuera posible encontrar soldados enfermos, quienes iban en las ancas de los jinetes" (Peñuela, 1919, p. 208). Uno de los testigos de la Campaña Libertadora, el edecán de Bolívar, Daniel Florencio O'Leary, plasmaba que atravesar el páramo de Pisba había dejado pérdidas incalculables para el Ejército Libertador, puesto que a su paso murieron grandes cantidades de personas, entre ellos, los extranjeros y los neogranadinos que no estaban acostumbrados a las bajas temperaturas (O’Leary 1989). Un tratamiento poco usual consistió en la flagelación, empleada con éxito para reanimar a los emparamados logrando así salvar algunos militares, como el caso de un coronel de caballería (Sotomayor Tribin, 1997, p. 189).

El 13 de julio, alejados del grueso de la división de vanguardia y retaguardia, el general de retaguardia ordenó a los jefes de las divisiones que

arreglaran del modo posible sus respectivos hospitales. Nombrara cada uno un oficial o sargento de mucha actividad, para que haga de contralor quienes recibían del proveedor general las raciones diariamente. Para mañana pasaran un estado de los muertos, heridos y dispersos (...) se ha ordenado al alcalde desocupe dos casas de las más grandes a fin de que no haya sino dos hospitales para poderlos asistir mejor. (Fundación para la Conmemoración del Bicentenario del Natalicio y el Sesquicentenario de la Muerte del General Francisco de Paula Santander, 1988, p. 24)

La misma fuente de consulta indica que "al llegar a Socha, las tropas estaban sin vestido, los hospitales llenos y el enemigo se encontraba a pocas jornadas" (p. 238). Pese a los infortunios que se les presentaron, Bolívar propició la organización de un hospital 
para que sirviera de refugio a los enfermos y así tuvieran un lugar en donde recuperarse. De igual forma ordenó a sus coroneles, en especial a Lara,

socorrer a los soldados que hayan quedado emparamados, sepultando los que indudablemente están muertos y sacando los que den alguna esperanza de vida. Debe usted saber en cuanto a esta última parte de comisión, que muchos días después de emparamados un hombre ha vuelto a la vida, por medio del calor y de los alimentos. (p. 246)

En julio de 1819, Bolívar dejó

al general Soublette entre Socha y Socotá activando el trabajo de las fraguas, que no descansaban en la faena de componer y reparar armas dañadas en el tránsito por el páramo y los combates recientes, así como también mirando por el cuidado de los hospitales, para que los enfermos se atendieran debidamente y los que se fueran restableciendo marcharan a incorporarse en sus filas respectivas (Peñuela, 1919, p. 277).

Sucedió igual con el coronel Rook, a quien se le encomendó, a su llegada a Socha, las diligencias pertinentes para trasladar el hospital a Socotá, dejando una guarnición de un poco más de 50 hombres (Rodríguez Plata \& Lee López, 1971, p. 415).

Si se tienen en cuenta las comunicaciones entre los oficiales, cumplir la misión no fue nada fácil. Con los pocos recursos obtenidos, debía llevarse a cabo la tarea logística y así dejar en reposo los enfermos más graves, pero en la ejecución de las acciones se encontraron con varias complicaciones para mover el hospital con la rapidez requerida. La solución fue trasladar una parte al pueblo de Socotá, mientras la otra debía esperar hasta que las condiciones fueran favorables (Rodríguez Plata \& Lee López, 1971, p. 131).

Después de la Batalla del Pantano de Vargas,

los señores Villates y Marińo proveyeron aquella noche y al día siguiente al aprovisionamiento del ejército, y los campesinos de todos los contornos acudieron con cuanto tenían, como también a recoger muchos heridos todavía y llevarlos a la casa, y reunir el botín de guerra que fue cuantioso. (Peñuela, 1919, p. 304)

También se ordenó que los heridos más graves fueran alojados en las casas de las familias más importantes de la región, para darles los cuidados necesarios y, así, seguir con las tropas (Peñuela, 1919, p. 305).

En los albores de la Batalla del Puente de Boyacá, la participación de las órdenes religiosas sirvió para la organización tanto de lugares como de medicamentos. Fray Ignacio Mariño, por ejemplo, participó activamente, lo cual mejoró la atención a los heridos y permitió "ayudar a bien morir a los caídos" (Mateus Caile, 1992, p. 41). Antes de su muerte, escribió en el testamento que dejaba tres tomos de materia médica y uno de rudimentos de medicina, los cuales se convirtieron en textos valiosos para la consulta de los nuevos médicos durante el siglo XIX. 


\section{Divisiones de vanguardia y retaguardia}

Después de identificar la organización de la Tercera División del bando realista comandada por el coronel José Barreiro, es pertinente analizar apartes de la configuración de las divisiones de vanguardia y retaguardia del Ejército patriota (tablas 2 y 3). La primera fue comandada por el general Francisco de Paula Santander y la segunda, por el general de brigada José Antonio Anzoátegui. Cabe resaltar que en esta última división, "más de 100 hombres del arma de infantería no pudieron combatir en los campos de batalla, su estado era lamentable y tuvieron que permanecer en el hospital" (Peńuela, 1919, p. 191).

Tabla 2. Estado Mayor General

\begin{tabular}{lc}
\hline \multicolumn{1}{c}{ Cargo } & Cantidad \\
\hline Jefe de operaciones & 1 \\
Oficiales & 8 \\
Secretario general & 1 \\
Médico & 1 \\
Asistente & 1 \\
\hline
\end{tabular}

Fuente: Peñuela (1919).

Tabla 3. Divisiones de vanguardia y retaguardia

\begin{tabular}{rc}
\hline Arma & Cantidades \\
\hline Infantería & 1116 \\
Caballería & 832 \\
\hline
\end{tabular}

Fuente: Thibaud (2003, pp. 301-302).

En comparación con las tablas 2 y 3 , la fuente mostró que en la organización del Estado Mayor General del Ejército patriota sí existió la figura del médico. Inclusive en las divisiones del cuerpo militar también existieron médicos, quienes hacían parte de la estructura jerárquica. Sin embargo, la búsqueda de más información arrojaría más datos que podrían complementar esta comparación. Un ejemplo puede ser determinar aproximadamente el personal médico con el cual contaba el Ejército Libertador frente al Ejército realista, teniendo en cuenta la cantidad de militares incorporados, porque de esta manera sabríamos si todo el personal de salud llevado a las campañas cumplió la labor de socorrer los heridos durante y después del combate. 


\section{Los primeros hospitales militares en el siglo XIX}

Desde finales del siglo XVIII, la Corona trató de instaurar un hospital militar que sirviera para la atención médica de los integrantes del Ejército. El intento más real en la Nueva Granada estuvo respaldado por el proyecto de creación de un hospital en Santafé, el cual fue materia de discusión entre 1790 y 1831 (Quevedo, 2008, p. 137). Sin embargo, en los tiempos iniciales del periodo republicano se trató de instaurar con más fuerza una institución al servicio médico militar.

Según las fuentes consultadas, en campaña los hospitales militares funcionaron como campamentos itinerantes que se movían junto con la tropa y servían para la atención de los soldados heridos o enfermos; ubicados en la retaguardia, defendidos por esta y junto con la intendencia del Ejército (Otálora Cascante, 2017). Ahí, el personal de salud o quien estuviera a cargo trataba de atender prontamente a los heridos.

En tiempos del general Sámano, el Convento de las Aguas sirvió como refugio a los militares enfermos o heridos por enfrentamientos. Para 1820, fue reabierto después de los inconvenientes presentados por las guerras independentistas, empezando a albergar los nuevos soldados patriotas, principalmente, a quienes quedaron en malas condiciones después de la Batalla de Boyacá.

La otra preocupación de los encargados de administrar esos lugares consistió en la adquisición de medicamentos suficientes y efectivos. Señala Emilio Quevedo que

... los listados de compras y recibos permiten establecer los medicamentos más usados, así como algunas sustancias que entraban en su composición: agua destilada, alcanfor, cuerno de ciervo, extracto de valeriana, amoniaco, opio, aceite de almendras, sal de Inglaterra, pez de Grecia, cardamomo, manteca de cacao, éter sulfúrico, extracto de Saturno, goma arábiga, maná, agua de rosas, carbonato de fierro, sulfato de quinina, linaza, trementina de frailejón, "raicilla de polvo", óxido blanco de zinc, nitrato de plata, y sanguijuelas. Estos remedios incluían productos naturales usados como alimentos, para la dietética, ocupaban un lugar importante en la terapéutica de la época, al lado de la farmacia y la cirugía. (Quevedo, 2008, p. 140)

En la época del doctor Juan Gualberto Gutiérrez, en el Régimen del Terror, cuando este era el jefe de hospital de Soatá y estaba al servicio del Ejército realista, solicitó un gran número de elementos al militar encargado para poder ejercer su profesión, parte de la lista y de las cantidades es la siguiente:

... manzanilla, flor de ídem, llantén, borraja, violetas, sanalotodo (bastante); upatoria (Sna juanito); raíces de rúchica (mucha); raíces de perejil, de hinojo, de espárrago y de achicoria; jumaria, parietaria, apio, eneldo, yerba de bubas, guasgüin, yerbabuena, espadilla, cebada (una carga); raíz de China (una carga); zarza (una carga); cilantro, calaguaba, polipodio, viravira, rosa de Alejandría, rosa de Castilla, pimpinela, toronjil, raíz y flores de malvavisco, quina (una carga); suelda con suelda y romero. (Rojas, 1940, p. 10) 
Según el proyecto del hospital, este debía contar con el siguiente personal: mayordomo, médico, cirujano, capellán, boticario, ropero, cocinero, sirvientas, practicantes y auxiliares — cabos de sala y asistentes- (Rojas, 1940, p. 140). Cada uno tenía sus funciones establecidas y era el personal mínimo para poner en marcha la atención hospitalaria.

Igual en la Grecia y Roma clásicas, los hospitales cobraron una gran importancia, tanto respecto de las enfermedades naturales como de aquellas dolencias producidas por acciones humanas en el contexto de guerra. De acuerdo con Gargantilla, la asistencia médica en Grecia se llevaba a cabo en espacios llamados iatreion, dedicados al cuidado de los enfermos, ambientes parecidos a las clínicas privadas. En Roma, los médicos atendían a los enfermos en las tabernae o tiendas, ubicadas en varios sectores de la ciudad. Cuando las ciudades se encontraban en guerra, existían los hospitales de campaña. En la novela histórica titulada Yo, Julia (Posteguillo, 2018), el médico Galeno acompañó y brindó tratamiento a las tropas aliadas de Septimio Severo en contra de Clodio Albino por el control de la Galia en el año 194 d. C.

\section{Conclusiones}

La medicina clásica griega del siglo $\mathrm{V}$ a. C. y romana del siglo I d. C. perduró por varias épocas, lo cual permitió el desarrollo de las civilizaciones. Los fundamentos hipocráticos y galenos fueron la base "técnica" y luego "científica" de la medicina moderna del siglo XIX, ejemplificada en esta ocasión por los primeros años del nacimiento de los procesos independentistas en América, en especial, el de la República de la Nueva Granada.

Los factores más determinantes en esa continuidad de la medicina clásica a la medicina moderna son el diagnóstico, el tratamiento y la relación de "amistad" entre el médico y el enfermo, actitud que se puede ver expresada en dos tipos: uno menos noble y otro más noble. Los médicos pertenecientes al primero practican su técnica movidos por un vehemente afán de prestigio y lucro. Los médicos del segundo tipo son técnicos profesionales y hombres sensibles al conocimiento del alma humana por encima del poder del dinero, sus actuaciones están fundamentadas en el amor por la naturaleza y por el arte de sanar (Laín Entralgo, 1964, p. 100). Precisamente en la Grecia clásica la salud significaba equilibrio de las potencias y armonía entre el hombre y la naturaleza.

Durante el desarrollo de la Campaña Libertadora, principalmente en tierras boyacenses, la sanidad militar se encargó de cuidar las enfermedades y heridas de los combatientes, buscar recursos económicos que facilitaran de alguna forma la supervivencia de la mayoría de militares y, por último, instalar en lugares alejados de los campos de batalla hospitales con la mínima capacidad de atención médica. La medicina fue ejercida en algunos casos por personaje ilustres de la sociedad neogranadina, quienes en su mayoría prestaron sus servicios a la causa independentista.

Los tratamientos médicos utilizados en aquella época —en algunas ocasioneseran de origen natural, basados en las costumbres y tradiciones de la Antigüedad clásica. El arte de curar todavía seguía siendo técnico (como en la Grecia y Roma antiguas), solo 
el Plan de Estudios de Medicina escrito por Mutis en el Colegio del Rosario y algunas enseñanzas de las teorías francesas fueron los soportes teóricos-científicos más relevantes; por tanto, los tratamientos y procedimientos médicos hechos después de las batallas fueron de significativa importancia, pues de ellos dependía la posibilidad salvar vidas y, gracias a ellos, se evidenció la necesidad de reglamentar la práctica médica.

La Campaña Libertadora tuvo grandes complicaciones a la hora de implantar un "proyecto" de sanidad militar. Los largos recorridos, la geografía del terreno y los cambios climáticos dificultaron en cierta medida que los comandantes de la divisiones, tanto del Ejército del rey como del Ejército patriota, pudieran desarrollar una logística adecuada en salvaguarda de sus militares. Pese a ello, se trató de instaurar improvisados hospitales de campaña que sirvieran como refugio a los enfermos y heridos.

En el mundo de las corrientes historiográficas, la historia de la medicina, los tratamientos, las enfermedades, los primeros médicos y la sanidad militar -independientemente de la directa relación con la historia militar y en el marco de la historia positivista - juegan un papel preponderante en la llamada Nueva Historia, porque se abre la puerta para nuevas investigaciones, basadas en un enfoque más interpretativo y analítico, sin descuidar el trabajo de documentos de archivo.

\section{Agradecimientos}

La autora desea agradecer a la Escuela Militar de Cadetes "General José María Córdova" por su apoyo en la realización de este artículo.

\section{Declaración de divulgación}

La autora declara que no existe ningún potencial conflicto de interés relacionado con la publicación del artículo. Este documento hace parte del proyecto de investigación "El Ejército en las Constituciones Federales 1853-1863: aproximación a un análisis comparativo”.

\section{Financiamiento}

La autora no declara fuente de financiamiento para la realización de este artículo.

\section{Sobre la autora}

Erika Constanza Figueroa Pedreros es magíster en Historia del Mundo Hispánico: Las Independencias en el Mundo Iberoamericano, Universidad Jaume I. Candidata a magíster en Historia Militar, de la Escuela Militar de Cadetes "General José María Córdova". Especialista en Docencia Universitaria. Historiadora de la Universidad Industrial de Santander y experta en Gestión y Conservación del Patrimonio Documental, de la Universidad Internacional de Andalucía.

https://orcid.org/0000-0001-6241-344X - Contacto: erika.figueroa@esmic.edu.co 


\section{Referencias}

Díaz Piedrahita, S., \& Mantilla, L. C. (2002). La terapéutica en el Nuevo Reino de Granada. Un recetario franciscano del siglo XVIII. Bogotá, Colombia: Academia Colombiana de Ciencias Exactas, Físicas y Naturales.

Earle, R. (2014). España y la Independencia de Colombia. Bogotá, Colombia: Ediciones Uniandes.

Fundación para la Conmemoración del Bicentenario del Natalicio y el Sesquicentenario de la Muerte del General Francisco de Paula Santander. (1988). Diarios de campaña, libros de órdenes y reglamentos militares 1818-1834. Bogotá, Colombia: Presidencia de la República.

Gargantilla, P. (2011). Breve historia de la medicina. Del chamán a la gripe. Madrid, España: Ediciones Nowtilus. Gozalbes Cravioto, E., \& García García, I. (2010). En torno a la medicina romana. Hispania Antigua, 33-34, 323-336.

Laín Entralgo, P. (1964). La relación médico-enfermo. Madrid, España: Ediciones Castilla S.A.

Laín Entralgo, P. (1970). La medicina hipocrática. Madrid, España: Ediciones Revista de Occidente.

Laín Entralgo, P. (1978). Historia de la medicina. Barcelona, Espańa: Salvat Editores.

Mateus Caile, M. (1992). Fray Ignacio Mariño, capellán del Ejército Libertador. Tunja, Colombia: Universidad Pedagógica y Tecnológica de Colombia.

Miranda Canal, N. (1992). La medicina en Colombia. De la influencia francesa a la norteamericana. Revista Credencial Historia, 29, 25-36.

O’Leary, D. F. (1989). Memorias. Bogotá: Ministerio de Educación Nacional.

Otálora Cascante, A. R. (2010). Por la salud de la nación. Las condiciones de salud de los ejércitos del rey y libertador en Costa Firme y Nueva Granada (Tesis de maestría). Universidad Nacional de Colombia, Bogotá, Colombia.

Otálora Cascante, A. R. (2017). Bajo las alas del Cóndor. La salud de los ejércitos del rey y libertador en el Virreinato de la Nueva Granada 1815-1820. Bogotá, Colombia: Universidad Nacional de Colombia.

Peñuela, C. L. (1919). Álbum de Boyacá. La campańa de 1819 (Vol. I). Bogotá, Colombia: Arboleda y Valencia. Posteguillo, S. (2018). Yo, Julia. Barcelona, España: Editorial Planeta.

Quevedo, E. (2008). De la medicina ilustrada a la medicina anatomoclinica (1782-1865) (Vol. II). Bogotá, Colombia: Tecnoquímicas.

Rodríguez Plata, H., \& Lee López, A. (1971). Documentos sobre la Campaña Libertadora de 1819 (Vol. II). Bogotá, Colombia: Editorial Andes.

Rojas, U. (1940). El profesor doctor Juan Gualberto Gutiérrez. Médico del precursor general Antonio Nariño y del Ejército Libertador de la Nueva Granada. Tunja, Colombia: Imprenta Oficial.

Romero, A. (1996). Historia de la medicina colombiana. Siglo XIX. Medellín, Colombia: Universidad de Antioquia.

Roselli, H. (1979). La medicina en la Independencia de Colombia. Revista Medicina. Órgano Informativo de la Academia Nacional de Medicina de Colombia, 2(2), 55-72.

Sanidad militar. (2001). En Diccionario de la Lengua Española (Vol. 22). Madrid, España: Espasa.

Sotomayor Tribin, H. A. (1997). Guerras, enfermedades y médicos en Colombia. Bogotá, Colombia: Escuela de medicina Juan N Corpas.

Thibaud, C. (2003). República en armas. Los ejércitos bolivarianos en la guerra de la independencia en Colombia y Venezuela. Bogotá, Colombia: Editorial Planeta. 